\title{
Development of nanostructured bioplastic material for wound healing
}

\author{
Ilmira R. Gilmutdinova, Elena Kostromina, Regina D. Yakupova, Petr S. Eremin \\ National Medical Research Center for Rehabilitation and Balneology \\ of the Ministry of Health of the Russian Federation, Moscow, Russian Federation \\ This article is distributed under the terms of the Creative Commons Attribution Noncommercial License (CC BY-NC 4.0) which permits \\ any noncommercial use, distribution, and reproduction in any medium, provided the original author(s) and source are credited.
}

\begin{abstract}
The development of new biomaterials whose characteristics are as close as possible to the properties of living human tissues is one of the most promising areas of regenerative medicine. This work aimed at creating a bioplastic material based on collagen, elastin and hyaluronic acid and studying its structure and properties to assess the prospects for further use in clinical practice. Bioplastic material was obtained by mixing collagen, hyaluronic acid and elastin in predetermined proportions with distilled water. We treated the material with photochemical crosslinking to stabilize biofilm in a liquid medium and form a nanostructured scaffold. A commercial human skin fibroblast cell culture was used to assess the biomaterial cytotoxicity and biocompatibility. The visualization and studies of the biomaterial structure were performed using light and scanning electron microscopy. It has been shown that the obtained biomaterial is characterized by high resilience; it has also a high porosity. The co-culturing of the bioplastic material and human fibroblasts did not reveal any of its cytotoxic effects on cells in culture. It was shown that the biomaterial samples could maintain physical properties in the culture medium for more than 10 days, while the destruction of the matrix was observed 3-4 weeks after the beginning of incubation. Thus, the created biomaterial can be used on damaged skin areas due to its physical properties and structure. The use of the developed biomaterial provides effective conditions for good cell proliferation, which allows us to consider it as a promising wound cover for use in clinical practice.
\end{abstract}

Key Words: bioplastic material, cell technology, tissue engineering, collagen, human fibroblasts

Eur J Transl Myol 2021; 31 (1): 9388. doi: 10.4081/ejtm.2021.9388

The conservative treatment of wounds of various etiologies does not always give the expected results. ${ }^{1,2}$ In this regard, the development of new modern wound dressings that contribute to the effective treatment and regeneration of the skin is required. ${ }^{3,4}$ Adhesiveness, plasticity, biocompatibility and maximum closeness in terms of fibroarchitectonics to body tissues and biological activity are important requirements for their creation, which allows achieving certain pharmaceutical effects from their use. Polymers (natural and synthetic), metals, inorganic materials, carbon-based materials and composites based on them are used to obtain bioplastic materials. ${ }^{5,6}$ Polymeric materials can be divided into natural and synthetic polymers. Natural polymers can be proteins (for example, collagen and fibrin), polysaccharides (chitosan, alginate, dextran etc.), synthetic polyvinyl alcohol, polyesters, polyphosphazenes, polyanhydrides, etc. ${ }^{7,8}$
Currently, in the development of implantable materials for regenerative medicine, preference is given to polymers of natural origin (biopolymers) and their derivatives. ${ }^{9,10}$ During implantation, they are split into simpler compounds that are excreted from the body or participate in metabolism at the cellular level. The advantage of biopolymers is that toxic products are not formed during their degradation. ${ }^{11}$ Composite matrices that include several complementary components to obtain systems with optimal properties (porosity, swelling, elasticity, mechanical strength) have been developed. Among these products, special attention is paid to materials based on collagen and hyaluronic acid - the main components of the extracellular matrix that are involved in the restoration of damaged structures of the dermis. ${ }^{12,13}$ This work aimed to create a new bioplastic material based on collagen, elastin and hyaluronic acid and study its structure and properties to assess the prospects for further use in clinical practice. 


\section{Nanostructured bioplastic material for wound healing}

Eur J Transl Myol 2021; 31 (1): 9388. doi: 10.4081/ejtm.2021.9388

\section{Materials and methods}

\section{Production of bioplastic material}

Bioplastic material was produced using the following components: collagen, hyaluronic acid and elastin, mixed in a proportion equal to that in an adult human dermis: collagen 70-90\%, hyaluronic acid 5-26\%, elastin $1-25 \%$. The material was subjected to photochemical crosslinking to stabilize biofilm in a liquid medium. The photochemical crosslinking was performed with an ultraviolet light source $(\lambda<260 \mathrm{~nm}$, $30 \mathrm{~cm}$ distance between UV source and biomaterial). In the process of material development, we were also elaborating on the technology of hydrogel production and settling the time of hydrogel exposure to UV, in order to form the photochemical crosslinking of a future biomaterial. Optimal conditions for obtaining the biomaterial were established by testing a series of experimental samples. Substances used for biomaterial production were mixed in the following proportions: collagen - 70\%, hyaluronic acid - $29 \%$ and elastin - $1 \%$. Distilled water was added, and stirred at medium/maximum speed for 5 - 10 minutes using a laboratory blender. The liquid was poured over iron and hydrophobic pallets. The liquid was left in a laminar cabinet under ultraviolet radiation for 6 hours with further keeping at room temperature for 18 hours.

Assessment of physical properties of bioplastic material The material was cut into samples of the same size (1 $\mathrm{cm} \times 1 \mathrm{~cm}$ ), placed in Petri dishes, immersed in $5 \mathrm{ml}$ of culture medium and visualized under an inverted microscope. Subsequently, the material samples were transferred to a $\mathrm{CO}_{2}$ incubator $\left(37^{\circ} \mathrm{C}, 5 \% \mathrm{CO}_{2}\right)$. Every 24 hours, the material specimens were weighed, and 10 $\mu \mathrm{l}$ of culture medium were selected to further study the change in optical density using a scanning spectrophotometer NanoDrop 2000C (Thermo Fisher Scientific, USA).

Assessment of cytotoxicity and biocompatibility of the bioplastic material

We studied the cytotoxicity of the bioplastic material on a commercial culture of human dermal fibroblasts (HDF) (Cell Applications, USA; Cat. \# 106K-05a). Cells were cultured in $\mathrm{CO}_{2}$ incubator $\left(37^{\circ} \mathrm{C}, 5 \% \mathrm{CO}_{2}\right)$ in Dulbecco's Modified Eagle's Medium (DMEM) with $4500 \mathrm{mg} / \mathrm{L}$ D-glucose (StemCell Technology, USA; Cat.\# 36250), with the addition of $10 \%$ of FBS (Biological Industries, Israel) and $2 \mathrm{mmol} / \mathrm{L}$ of Lglutamine (StemCell Technologies, USA; Cat.\# 07100). The culture of cells was passaged in a $d=94$ $\mathrm{mm}$ Petri dish, with $25 \times 10^{3}$ cells $/ \mathrm{cm}^{2}$ density and cultured according to standard technique up till the formation of a monolayer. After that, we sampled a supernatant, added a new medium, put in a biomaterial sample and left it in $\mathrm{CO}_{2}$ incubator for 72 hours. Upon expiry of this period, we sampled a supernatant and transferred it into a new Petri dish to count dead cells. We used Trypsin-EDTA, $0.25 \%$ (StemCell Technologies, USA; Catalog \#07901) to get cells from the surface of the studied Petri dishes. The Countess automated counter (Invitrogen, Korea) was used to count cells and assess their viability by the manufacturer's procedure. To study biocompatibility of the bioplastic material, we put $0.5 \mathrm{~cm}^{2}$ size samples into Petri dishes and added $1 \mathrm{ml}$ of cell suspension of HDF containing $12.5 \times 10^{3}$ cells in each, forming a "drop". After 30 minutes in $\mathrm{CO}_{2}$ incubator, the samples were added with a growth medium and cultured for 10 days. To assess the viability of cells, we performed staining with fluorescent dyes SYTO 9 (Invitrogen, USA) and propidium iodide (Sigma-Aldrich, USA). ${ }^{14}$ The
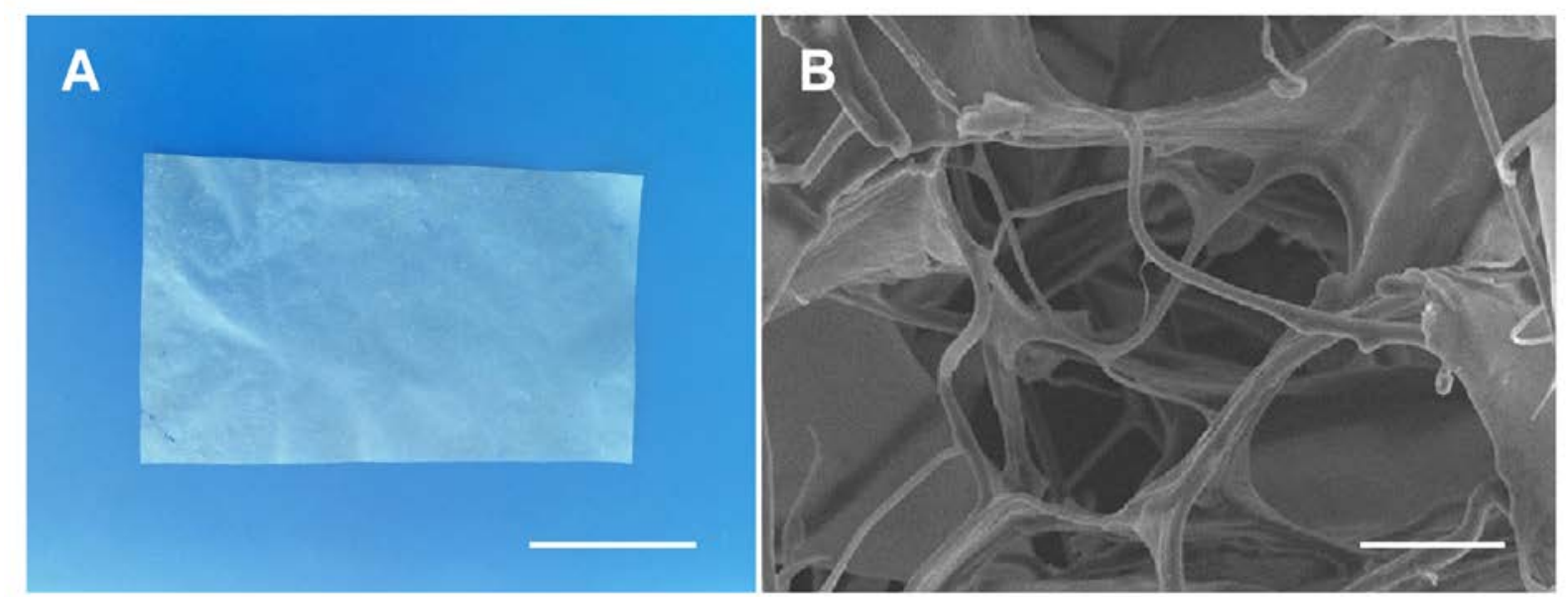

Fig 1. The appearance (A) of the bioplastic material and its structure (B). A, Scale bar $=5 \mathrm{~cm}$; B, Scanning electron microscopy. Scale bar $=50 \mu \mathrm{m}$ 


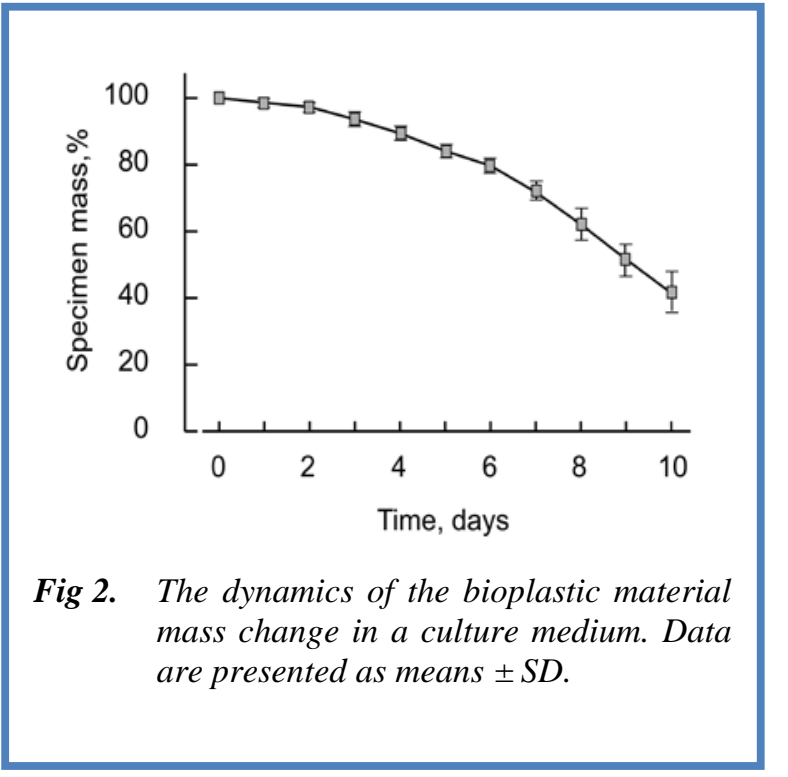

number of live and dead cells was determined according to the number of cell nuclei revealed by SYTO 9 and propidium iodide, respectively.

\section{Visualization of biomaterial samples}

Examination of samples of the matrix and cells attached to it was conducted using an inverted microscope Leica DM IL LED (Leica Microsystems, Germany) and a laser scanning confocal microscope LSM 510 META based on an inverted microscope Axiovert 200M (Carl Zeiss, Germany). Image analysis was perform using Zeiss 3D for LSM Version 1.4.2 software (Carl Zeiss, Germany). Scanning electron microscopy (SEM) was carried out using Camscan S2 microscope (Cambridge Instruments, $\mathrm{UK}$ ) in SEI mode with 10 -nm resolution and operating voltage of $15 \mathrm{kV}$.

\section{Statistical analysis}

The data are presented as means \pm SD. Statistical analysis was carried out using Student's t-test. The level of statistical significance was set at $\mathrm{P}<0.05$.

\section{Results \& Discussion}

We have developed a new bioplastic material based on collagen, hyaluronic acid and elastin and studied its properties. The resulting biomaterial is characterized by high elasticity and resilience, which allows its use on damaged skin areas with uneven relief or on mobile areas. The obtained material has also high porosity, with a pore diameter of 100-200 $\mu \mathrm{m}$ (Figure 1). Thus, the physical structure of bioplastic material allows for gas exchange of wound surface and exudates withdrawal. ${ }^{15}$ Taking into account that the obtained biomaterial can be used as a scaffold for cell culture, this structure can provide conditions for cell migration inside as well as homogeneity of cell culture conditions and mechanical properties of the structure. The study of matrix stability in culture medium revealed that this material can preserve its physical properties for more than 10 days (Figure 2). After 5 days, the biomaterial in the $\mathrm{CO}_{2}$ incubator degraded by 15-18\%. Complete destruction of the biomaterial occurred after 3-4 weeks of incubation. Co-culturing bioplastic material and cultures of human fibroblasts did not reveal any cytotoxic effects on cell cultures. After 72 hours of incubation, no squamous cells in a culture liquid and no changes in morphological parameters of fibroblasts on the plastic surface were observed. Furthermore, we registered a higher proliferative activity of the cells in the presence of material samples than one in the control group. It was noted that all cells attached to the surface within 4-6 hours after the start of incubation when
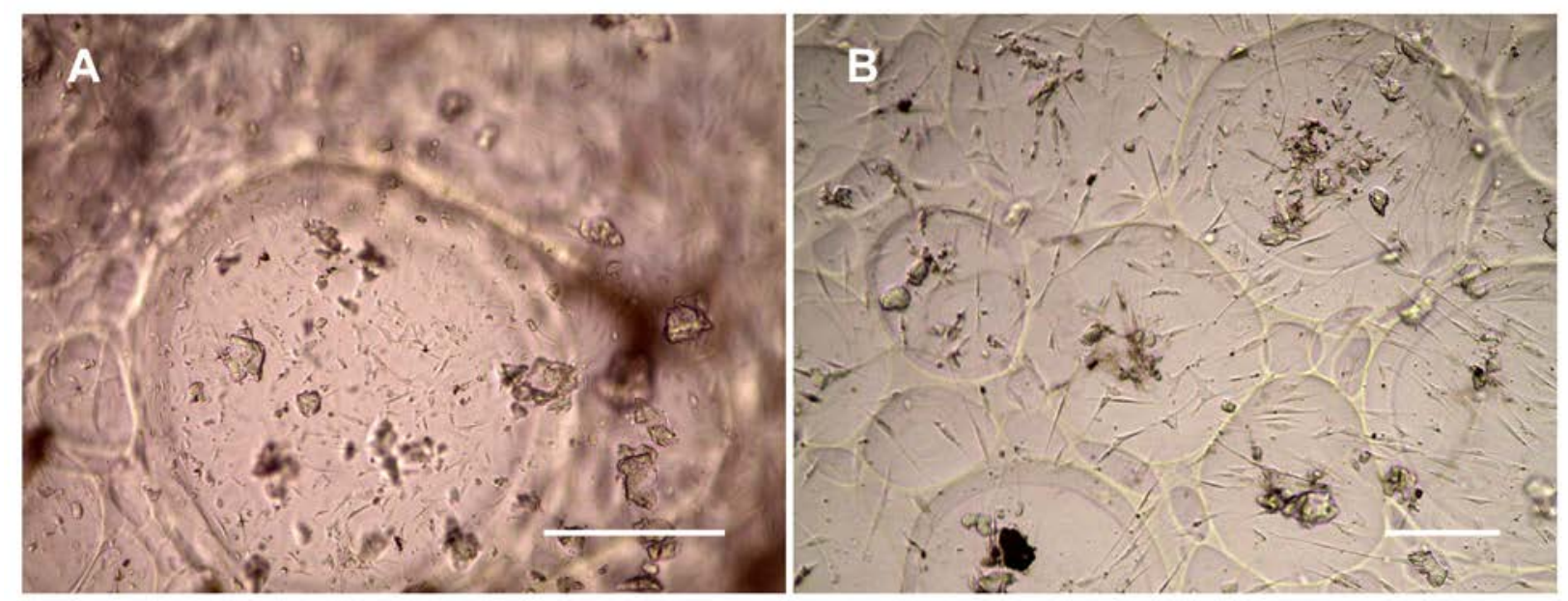

Fig 3. Biocompatibility (A) and cytotoxicity (B) studies of the bioplastic material. 5 days of culturing. Light microscopy. Scale bar $=100 \mu \mathrm{m}$. 


\section{Nanostructured bioplastic material for wound healing}

Eur J Transl Myol 2021; 31 (1): 9388. doi: 10.4081/ejtm.2021.9388

fibroblasts were cultured on the biomatrix. We have been culturing fibroblasts for 2 weeks to study the growth dynamics of cells in the matrix (Figure 3). Cell counts were made on the images of horizontal projections of a series of optical slices of the matrix of known thickness. The presence of dividing cells was detected already in 24 hours after attachment. The total number of cells per $\mathrm{mm}^{3}$ was $440 \pm 84(\mathrm{n}=10)$. In 5 days, the number of cells per $\mathrm{mm}^{3}$ increased to $776 \pm 134$ $(n=10, p<0.001)$. The maximum number of cells was observed on the 10th day after attachment, which was up to $2155 \pm 335$ cells per $\mathrm{mm}^{3}(\mathrm{n}=10, \mathrm{p}<0.001)$. During culturing on a flat surface, cell cultures of fibroblasts reached the monolayer on the 6th day. Due to its properties and structure, the developed biomaterial could have a wide range of clinical applications. This biomaterial can be applied as a mask or a patch. The material can also be potentially used as a carrier of drugs, e.g., antibacterial drugs, as well as agents that affect cell differentiation, proliferation, and interaction to produce viable cultures to replace lost tissue. This biomaterial seems promising for 3D bioprinting that will create three-dimensional structures with desirable biological, structural, and mechanical properties. There is an increasing demand for new biomaterials in 3D bioprinting. ${ }^{16}$ The main physical and chemical parameters determining the suitability of hydrogel are their rheological characteristics and cross-linking mechanisms. Materials used for 3D bioprinting must support cell viability and proper functioning, by providing structural and mechanical support for the model, biocompatibility, and easy manipulation of the bioprinter for acquisition of a 3D structure. ${ }^{17,18}$ As such, this biomaterial meets the major requirements for potential use with 3D printing. The effective solution for any existing problems, linked to technical and cellular aspects of this procedure, will make it possible to obtain samples of various tissues that can in turn be used in regenerative medicine.

In conclusion, the developed biomaterial can be used on damaged skin areas due to its physical properties and structure. The use of created wound cover provides effective conditions for good cell proliferation, which allows us to consider it as a promising biomaterial for use in clinical practice.

\section{List of acronyms}

DMEM - Dulbecco's Modified Eagle’s Medium

HDF - Human Dermal Fibroblasts

\section{Authors contributions}

IG, EK, RY, PE designed research; RY, PE performed experiments; IG, EK, RY, PE analyzed data; IG, EK, RY, PE wrote the paper. All authors approved the manuscript.

\section{Acknowledgments}

Funding This study was supported by the Foundation for Promoting Innovation (Grant \#14612GU/2019).

\section{Conflict of Interest}

The authors declare they have no financial, personal, or other conflicts of interest.

\section{Ethical Publication Statement}

We confirm that we have read the Journal's position on issues involved in ethical publication and affirm that this report is consistent with those guidelines.

\section{Corresponding Author}

Ilmira R. Gilmutdinova, National Medical Research Center for Rehabilitation and Balneology of the Ministry of Health of the Russian Federation Phone: +7 968 6861979. ORCID iD: 0000-0001-6743-2615

Email: gilm.ilmira@mail.ru

\section{Emails and ORCID iD of Coauthors}

Elena Kostromina: bioimed07@hotmail.com

ORCID iD: 0000-0002-9728-7938

Regina D. Yakupova: regina_yakupova.biotech@bk.ru

ORCID iD: 0000-0001-8514-2240

Petr S.Eremin: ereminps@gmail.com

ORCID iD: 0000-0001-8832-8470

\section{References}

1. Frykberg RG, Banks J. Challenges in the treatment of chronic wounds. Adv Wound Care (New Rochelle) 2015;4:560-82. doi.10.1089/wound. 2015.0635.

2. Xu J, Zheng S, Hu X, Li L, Li W, Parungao R, Wang Y, Nie Y, Liu T, Song K. Advances in the research of bioinks based on natural collagen, polysaccharide and their derivatives for skin 3d bioprinting. Polymers (Basel) 2020 May 29;12(6):1237. doi: 10.3390/polym12061237. doi: 10.3390/polym 12061237.

3. Kotenko K, Eremin I, Moroz B, Bushmanov AJu, Nadezhina NM, Galstjan IA; Grinakovskaja OS, Aksenenko AV, Deshevoj JuB, Lebedev VG, Slobodina TS, Zhgutov JuA, Lauk-Dubickij SE. Eremin PS. Cell technologies in the treatment of radiation burns: Experience burnasyan federal medical biophysical centre. Cellular Transplantation \& Tissue Engineering 2012;7:97102.

4. Tottoli EM, Dorati R, Genta I, Chiesa E, Pisani S, Conti B. Skin wound healing process and new emerging technologies for skin wound care and regeneration. Pharmaceutics 2020;12. doi.10.3390/ pharmaceutics12080735.

5. Sheikh Z, Najeeb S, Khurshid Z, Verma V, Rashid $\mathrm{H}$, Glogauer M. Biodegradable materials for bone repair and tissue engineering applications. Materials (Basel) 2015;8:5744-94. doi.10.3390/ma 8095273.

6. Shtil'man MI, Mezhuev YO. Implanted and unimplanted medical and biological polymers. 


\section{Nanostructured bioplastic material for wound healing}

Eur J Transl Myol 2021; 31 (1): 9388. doi: 10.4081/ejtm.2021.9388

Fibre Chemistry (English Translation of Khimicheskie Volokna) 2016;48:249-52.

7. Guan J, Sacks MS, Beckman EJ, Wagner WR. Synthesis, characterization, and cytocompatibility of elastomeric, biodegradable poly(esterurethane)ureas based on poly(caprolactone) and putrescine. J Biomed Mater Res 2002;61:493-503. doi.10.1002/jbm.10204.

8. Yu Z, Lili J, Tiezheng Z, Li S, Jianzhuang W, Haichao D, Kedong S, Tianqing L. Development of decellularized meniscus extracellular matrix and gelatin/chitosan scaffolds for meniscus tissue engineering. Biomed Mater Eng 2019;30:125-32. doi.10.3233/bme-191038.

9. Reis RL, Neves N, Mano JF, Gomes ME, Marques Ap. Natural-based polymers for biomedical applications. Natural-Based Polymers For Biomedical Applications. Woodhead Publishing Series in Biomaterials. 2008:XXIII-XXV.

10. Azimi B, Maleki H, Zavagna L, De la Ossa JG, Linari S, Lazzeri A, Danti S. Bio-based electrospun fibers for wound healing. J Funct Biomater 2020;11. doi.10.3390/jfb11030067.

11. Song R, Murphy M, Li C, Ting K, Soo C, Zheng Z. Current development of biodegradable polymeric materials for biomedical applications. Drug Des Devel Ther 2018;12:3117-45. doi.10.2147/DDDT.S165440.

12. Mao AS, Mooney DJ. Regenerative medicine: Current therapies and future directions. Proc Natl Acad Sci U S A. 2015;112:14452-9. doi: 10.1073/pnas.1508520112.
13. Carruthers CA, Dearth CL, Reing JE, Kramer CR, Gagne DH, Crapo PM, Garcia O Jr, Badhwar A, Scott JR, Badylak SF. Histologic characterization of acellular dermal matrices in a porcine model of tissue expander breast reconstruction. Tissue Eng Part A 2015;21:35-44. doi: 10.1089/ten.TEA. 2014.0095.

14. Haugland RP, Gregory J, Spence MTZ, Johnson I D. Handbook of fluorescent probes and research products: Molecular Probes. Pennsylvania State University. 2002.

15. Chantre CO, Gonzalez GM, Ahn S, Cera L, Campbell PH, Hoerstrup SP, Parker KK. ACS Appl Mater Interfaces. 2019 Dec 11;11(49):4549845510. doi: 10.1021/acsami.9b17322. Epub 2019 Nov 22.

16. Uludag H, Pandit A, Kuhn L. Editorial: Enabling biomaterials for new biomedical technologies and clcinical therapies. Front Bioeng Biotechnol 2020 Jun 5;8:559. doi: 10.3389/fbioe.2020.00559.

17. Tetsuka H, Shin SR. Materials and technical innovations in 3d printing in biomedical applications. J Mater Chem B 2020 Apr 21;8:29302950. doi: 10.1039/d0tb00034e.

18. Chan WW, Yeo DCL, Tan V, Singh S, Choudhury D, Naing MW. Additive biomanufacturing with collagen inks. Bioengineering (Basel) 2020 Jul 1;7:66. doi: 10.3390/bioengineering7030066

Submitted: October 6, 2020

Revision received: November 11, 2020 Accepted for publication: November 11, 2020 